Original

\title{
The effect of mesenchymal stem cells on osteoclast precursor cell differentiation
}

\author{
Takaharu Abe1), Keisuke Sumi1), Ryo Kunimatsu1), Nanae Oki1), Yuji Tsuka1), \\ Kengo Nakajima2), Kazuyo Ando'2), and Kotaro Tanimoto')
}

\author{
1)Department of Orthodontics, Division of Oral Health and Development, Hiroshima University Hospital, \\ Hiroshima, Japan \\ 2)Department of Orthodontics, Applied Life Sciences, \\ Hiroshima University Institute of Biomedical \& Health Sciences, Hiroshima, Japan
}

(Received September 5, 2017; Accepted January 30, 2018)

\begin{abstract}
Transplantation of mesenchymal stem cells (MSCs) has been extensively studied in the field of regenerative medicine. Bone regeneration is achieved via the interaction of osteoblasts and osteoclasts. However, the influence of MSCs on osteoclasts is unknown. The purpose of this study was to investigate the effect of MSCs on the expression of genes for osteoclast differentiation factors using qPCR after indirect co-culture of MSCs and RAW264 cells. The numbers of osteoclasts after addition of soluble receptor activator of nuclear factor kappa $B$ (NF-кB) ligand (sRANKL) were also compared. Expression of osteoprotegerin (OPG) by MSCs was significantly elevated in co-culture over time. The differentiation of RAW264 cells into mature osteoclasts following addition of sRANKL was significantly inhibited by co-culture with MSCs. Expression of RANK, colony stimulating factor 1 receptor, $N F-\kappa B$, and nuclear factor of activated T-cell cytoplasmic 1 in RAW264 cells was significantly inhibited by co-culture with MSCs. Expression of OPG protein was higher in co-culture with RAW264 cells than in MSCs alone, and the expression level was clearly higher than that
\end{abstract}

Correspondence to Dr. Ryo Kunimatsu, Department of Orthodontics, Division of Oral Health and Development, Hiroshima University Hospital, 1-2-3, Kasumi, Minami-ku, Hiroshima 734-8553, Japan

Fax: +81-82-257-5687 E-mail: ryoukunimatu@hiroshima-u.ac.jp

J-STAGE Advance Publication: December 11, 2018

Color figures can be viewed in the online issue at J-STAGE.

doi.org/10.2334/josnusd.17-0315

DN/JST.JSTAGE/josnusd/17-0315 of RANKL. MSCs appeared to inhibit osteoclast differentiation via expression of OPG.

Keywords: mesenchymal stem cell; osteoclast precursor cell; differentiation; bone regeneration.

\section{Introduction}

Cleft lip and palate is a congenital condition characterized by jaw clefts due to failure of the palatal shelves to fuse properly. In the Japanese population its incidence is relatively high at $0.10 \%(1)$. In most cases, continuity of the dentition is lost due to the jaw cleft, and therefore transplantation of autologous iliac bone is mainly employed to repair the defect (2). However, the surgical procedures involved are highly invasive and stressful (3). For these reasons, it has been proposed that bone regeneration therapy using mesenchymal stem cells (MSCs) might reduce the degree of surgical invasiveness and stress burden on affected patients.

In a previous study, bone was successfully regenerated following transplantation of MSCs and a carbonated hydroxyapatite scaffold into artificial bone defects in beagle dogs. For bone regeneration in a jaw cleft, teeth must be able to erupt, and the regenerating bone must be amenable to orthodontic treatment. Bone regeneration in the artificial jaw cleft was confirmed, and orthodontic tooth movement to the bone regeneration site was performed. At the site of MSC transplantation, carbonated hydroxyapatite was absorbed early and replaced with regenerated bone $(4,5)$. It is desirable for the implanted carrier to be absorbed at an early stage, followed by rapid 
bone regeneration. This would involve absorption by osteoclasts and subsequent replacement with bone by osteoblasts.

Osteoclasts are multinucleated giant cells with eosinophilic cytoplasm and tartrate-resistant acid phosphatase (TRAP) activity $(6,7)$. Osteoblasts and their progenitor cells express cytokines involved in osteoclastogenesis, such as membrane-bound protein receptor activator of nuclear factor kappa B (NF-kB) ligand (RANKL) and osteoprotegerin (OPG), which acts as a RANKL decoy receptor to inhibit osteoclastogenesis, and macrophage colony-stimulating factor (M-CSF). In the presence of these cytokines, hematopoietic stem cells differentiate into osteoclasts $(8,9)$. Binding of RANKL to receptor activator of NF-kB (RANK) and M-CSF to colony stimulating factor 1 receptor (CSF1R) on osteoclast precursors results in activation of $\mathrm{NF}-\mathrm{\kappa B}$ and nuclear factor of activated T-cell cytoplasmic 1 (NFATc1) (10). Thus, maintenance of balanced and normal bone remodeling requires signaling via cytokine-mediated interactions or cell-cell contact. Although the relationship between osteoblasts and spleen cells has been studied by Takahashi et al. (11), few have investigated the involvement of transplanted MSCs in osteoclast differentiation.

Although intercellular contact has been reported to be important (11-14), the mechanism responsible for paracrine information transmission has remained unknown. In the present study, therefore, the effect of MSCs on the expression of osteoclast differentiation factors in RAW264 cells was examined by co-culturing them with MSCs.

\section{Materials and Methods}

\section{Cell culture}

Balb/c mouse bone marrow-derived MSCs (Cyagen, Santa Clara, CA, USA) and the Balb/c mouse osteoclast precursor RAW264 cell line (Riken Cell Bank no. RCB 0535, RIKEN, Tokyo, Japan) were used. MSCs and RAW264 cells were cultured in $\alpha$-minimum essential medium ( $\alpha$-MEM; Sigma Aldrich, St. Louis, MO, USA) supplemented with $10 \%$ fetal bovine serum (Biological Industries, Hartford, CT, USA), 10\% sodium bicarbonate, and $0.7 \mathrm{mg} / \mathrm{mL} L$-glutamine. All cultures were incubated at $37^{\circ} \mathrm{C}$ in a humidified atmosphere with $5 \% \mathrm{CO}_{2}$.

\section{Time course of expression of osteoclast differentiation-related genes in MSCs}

MSCs were seeded at $4.8 \times 10^{4}$ cells/well in 6-well plates (Falcon, Corning, NY, USA) and cultured in $\alpha$-MEM (Sigma Aldrich) supplemented with $10 \%$ fetal bovine serum (Biological Industries), $0.06 \mu \mathrm{g} / \mathrm{mL}$ kanamycin
Table 1 RCR primer sequences (mouse)

\begin{tabular}{lll}
\hline Gene & & Sequence $\left(5^{\prime} \rightarrow 3^{\prime}\right)$ \\
\hline GAPDH & Forward & ATGGCCTTCCGTGTTCCT \\
& Reverse & CCCAAGATGCCCTTCAGT \\
RANKL & Forward & CATCGCTCTGTTCCTGACTTC \\
& Reverse & AGGAGTCAGGTAGTGTGTCTTCA \\
M-CSF & Forward & GACCCTCGAGTCAACAGAGC \\
& Reverse & TGTCAGTCTCTGCCTGGATG \\
OPG & Forward & AAAGTGAATGCCGAGAGTGTAG \\
& Reverse & GCTTTCACAGAGGTCAATGTC \\
VEGF & Forward & ATGCGGATCAAACCTCA \\
& Reverse & TTCTGGCTTTGTTCTGTCTT \\
RANK & Forward & CGAGGAAGATTCCCACAGAG \\
& Reverse & CAGTGAAGTCACAGCCCTCA \\
CSF1R & Forward & GACCTGCTCCACTTCTCCAG \\
& Reverse & GATGTCCCTAGCCAGTCCAA \\
NF-кB & Forward & CACCTAGCTGCCAAAGAAGG \\
& Reverse & GCAGGCTATTGCTCATCACA \\
NFATC1 1 & Forward & ACCACCTTTCCGCAACCA \\
& Reverse & TTCCGTTTCCCGTTGCA \\
\hline
\end{tabular}

(Meiji, Tokyo, Japan), 5 units/mL penicillin (Meiji), and $125 \mu \mathrm{g} / \mathrm{mL}$ amphotericin B (MP Biomedicals, Santa Ana, $\mathrm{CA}, \mathrm{USA}$ ) at $37^{\circ} \mathrm{C}$ in $5 \% \mathrm{CO}_{2}$. At $4,6,8$, and 10 days from the start of culture, the cells were collected, total RNA was extracted and purified using $\beta$-mercaptoethanol (Sigma-Aldrich Japan, Tokyo, Japan), and a Simpli Amp Thermal Cycler (Applied Biosystems, Foster City, CA, USA) was used to synthesize cDNA. Using the prepared cDNA as a template, expression of the osteoclast differentiation factor, RANKL; M-CSF; the osteoclast differentiation inhibitor, OPG; and vascular endothelial cell growth factor (VEGF) in MSCs was compared using the SYBR Green Real-time PCR Master Mix (Toyobo, Osaka, Japan) and Light Cycler 480 II (Roche Diagnostics, Basel, Switzerland). The primers used are shown in Table 1. Reaction conditions were 40 cycles with denaturation at $95^{\circ} \mathrm{C}$ for $15 \mathrm{~s}$, annealing at $55^{\circ} \mathrm{C}$ for $30 \mathrm{~s}$, and extension at $72^{\circ} \mathrm{C}$ for $10 \mathrm{~s}$.

\section{Effect of MSC co-culture on osteoclast differentiation of RAW264 cells}

RAW264 cells were seeded in 24-well plates (Falcon) at $5 \times 10^{3}$ cells/well. On the following day (day 0 ), RANK Ligand, Soluble, Murine, Recombinant (sRANKL; Pepro Tech, Rocky Hill, CT, USA) was added to $\alpha$-MEM (Sigma Aldrich) at $100 \mathrm{ng} / \mathrm{mL}$. The medium was replaced with medium containing sRANKL every 2 nd day. On day 8, TRAP staining was performed using a TRAP/ALP staining kit (Wako, Tokyo, Japan) to confirm differentiation into osteoclasts, and cells were observed with a DMil microscope (Leica, Wetzlar, Germany).

Next, MSCs were seeded into 6-well plates (Falcon) at $1 \times 10^{4}$ cells/well, and RAW264 cells were seeded at 
$5 \times 10^{3}$ cells/well in Cell Culture Inserts High Density, Translucent PET Membrane 24-well plates, $0.4 \mu \mathrm{m}$ pore size (Falcon) for indirect co-culture. In other 6-well plates (Falcon), RAW264 cells were cultured alone at $5 \times$ $10^{3}$ cells/well. On the following day (day 0 ), the culture medium was replaced with medium containing sRANKL (Pepro Tech) (sRANKL was added to both RAW264 only cultures and RAW264/MSC co-cultures), and the medium was replaced with fresh medium every 2 nd day. From day 0 (at the start of sRANKL addition), using the number of actin ring-forming cells with five or more nuclei to represent the number of mature osteoclasts, osteoclast counts among RAW264 cells cultured alone and in RAW264 cell/MSC co-cultures were compared.

\section{Effect of MSC co-culture on expression of osteoclast differentiation-related genes in RAW264 cells}

MSCs were seeded at $4.8 \times 10^{4}$ cells/well in Cell Culture Inserts, 6 -well plates, $0.4 \mu \mathrm{m}$ pore size (Falcon), RAW264 cells were seeded in these 6-well plates (Falcon) at 2.4 $\times 10^{4}$ cells/well, and indirect co-culture was performed. When confluent on day 8, RAW264 cells were collected. Using qPCR, gene expression in RAW264 cells cultured alone and co-cultured indirectly with MSCs was compared. Expression of the following genes was compared: RANK, which is a receptor for RANKL; CSF1R, which is a receptor for M-CSF; NF- $\mathrm{KB}$; and NFATc1, which is an osteoclast precursor intracellular transcription factor. The primers used are shown in Table 1.

\section{Time course of expression of osteoclast} differentiation-related genes in co-cultures of MSCs and RAW264 cells

RAW264 cells were seeded at $2.4 \times 10^{4}$ cells/well in Cell Culture Inserts Transparent PET Membrane, 6-well plates, $0.4 \mu \mathrm{m}$ pore size (Falcon), MSCs were seeded in these 6-well plates (Falcon) at $4.8 \times 10^{4}$ cells/well, and indirect co-culture was performed. A comparative study was carried out with qPCR to analyze the expression of RANKL, M-CSF, OPG, and VEGF at 4, 6, 8, and 10 days from the start of co-culture.

\section{Amount of RANKL and OPG protein secreted into co-cultures of MSCs and RAW264 cells}

MSCs were seeded into 6-well plates (Falcon) at $4.8 \times 10^{4}$ cells/well, RAW264 cells were seeded at $2.4 \times 10^{4}$ cells/ well in Cell Culture Inserts, 6-well plates, $0.4 \mu \mathrm{m}$ pore size (Falcon), and indirect co-culture was performed. On day 8 , the medium was changed to serum-free medium, and after $24 \mathrm{~h}$, the conditioned medium was collected and centrifuged in Amicon Ultra 15-mL Centrifugal Filters
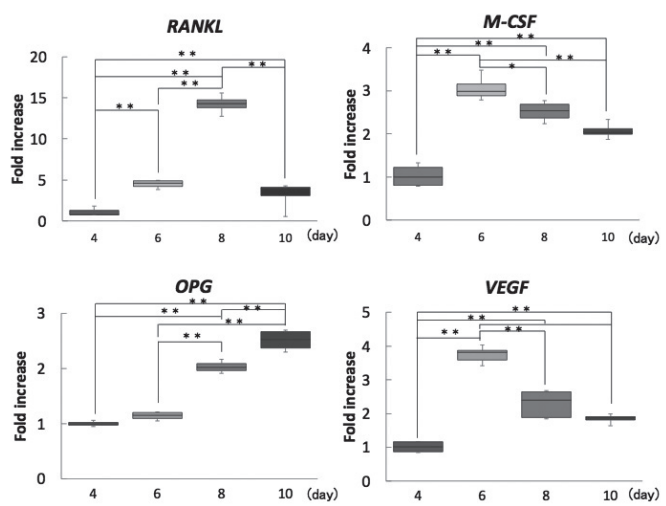

Fig. 1 Time course of expression of osteoclast differentiationrelated genes in MSCs.

(Millipore Sigma, Frankfurt, Germany) to obtain 5-foldconcentrated supernatant. ELISA was performed using the Mouse TRANCE/RANK L/TNFSF11 Quantikine ELISA Kit (R\&D Systems Bio-Techne, Minneapolis, MN, USA) and the Mouse Osteoprotegerin/TNFRSF11B Quantikine ELISA Kit (R\&D Systems Bio-Techne) with RANKL and OPG.

\section{Statistical analysis}

Multiple comparison tests were performed with the Bonferroni/Dunn method, and comparative tests between two groups were performed with the Mann-Whitney $U$ test. Results are shown as median and interquartile range. Differences at $P<0.05$ were considered statistically significant.

\section{Results}

Time course of expression of osteoclast differentiation-related genes in MSCs

In MSCs, the expression of M-CSF and VEGF peaked on day 6 , followed by a significant reduction (Fig. 1). A significant increase in RANKL was observed up to day 8 , but on day 10 it was significantly decreased. Expression of OPG was significantly $(P<0.01)$ elevated from day 4 to day 10. Relative to that on day 4 , the expression level of RANKL on day 8 was increased about 14 -fold, that of M-CSF on day 6 was increased about 3 -fold, that of OPG on day 10 was increased about 2.5 -fold, and that of VEGF on day 6 was increased about 3.7-fold.

\section{Effect of MSC co-culture on osteoclast differentiation of RAW264 cells}

After addition of sRANKL to RAW264 cells, differentiation into osteoclasts was confirmed by TRAP staining on day 8 (Fig. 2A and 2B).

When RAW264 cells were cultured alone, osteoclast differentiation was observed 2 days after sRANKL addition, whereas in indirect co-culture with MSCs, no 


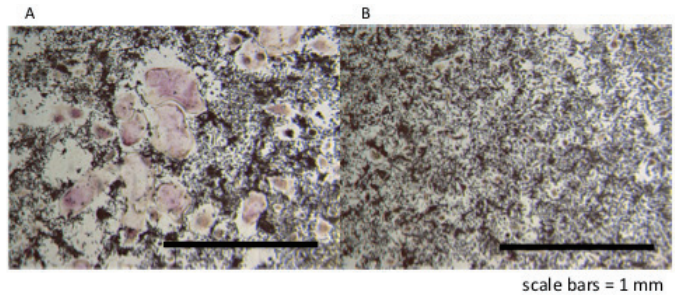

Fig. 2 A: RAW264 cells treated with sRANKL differentiated into osteoclast-like cells, as demonstrated by TRAP staining. B: RA264 cells treated with sRANKL and subjected to TRAP staining at day 8 .

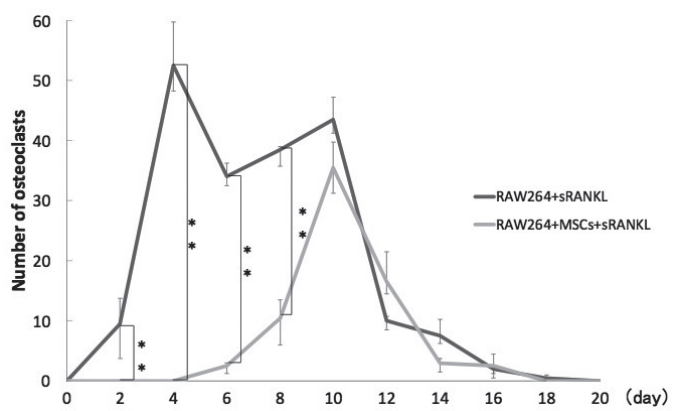

Fig. 3 Effect of MSC co-culture on osteoclast differentiation of RAW264 cells.
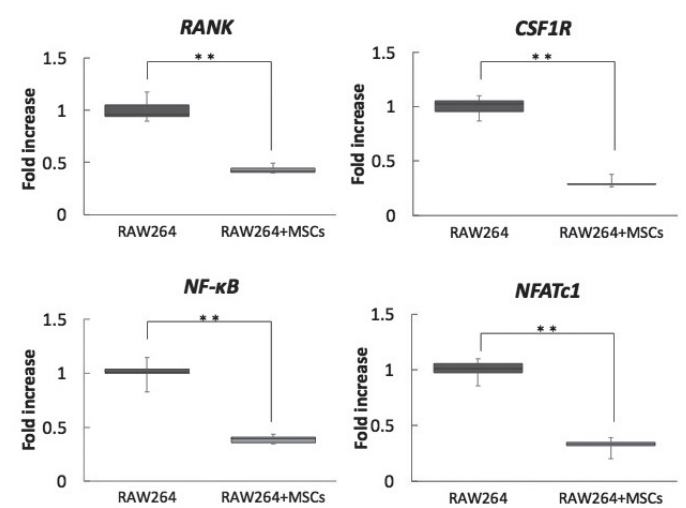

Fig. 4 Effect of MSC co-culture on osteoclast differentiationrelated gene expression in RAW264 cells on day 8 .

osteoclast differentiation was observed at 2 and 4 days (Fig. 3). Differentiation was observed to begin on day 6 in regions outside those directly below the cell culture insert. From day 2 to 8 , the number of differentiated osteoclasts was significantly lower in indirect co-culture than in cultures of RAW264 cells alone. No significant difference in osteoclast survival was evident between the two groups. Incidentally, when sRANKL was not added, osteoclast differentiation was not observed in indirect co-culture with MSCs.

\section{Effect of MSC co-culture on expression of osteoclast differentiation-related genes in RAW264 cells} On day 8, expression of RANK, CSF1R, NF-kB, and NFATc1 in RAW264 cells co-cultured with MSCs was
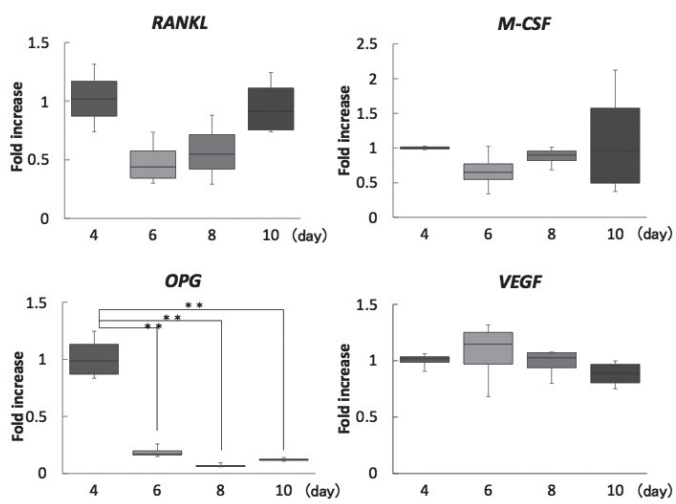

Fig. 5 Time course of expression of osteoclast differentiationrelated genes during co-culture of MSCs and RAW264 cells.
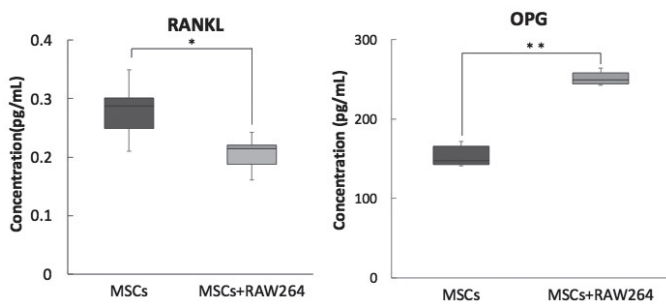

Fig. 6 Amounts of RANKL and OPG secreted into the medium on day 8 of co-culture of MSCs and RAW264 cells.

significantly $(P<0.01)$ suppressed compared to that in RAW264 cells cultured alone (Fig. 4). Expression levels of all four genes in RAW264 cells co-cultured with MSCs were less than half of those in RAW264 cells cultured alone.

\section{Time course of expression of osteoclast} differentiation-related genes in co-cultures of MSCs and RAW264 cells

No significant change was seen in the expression of RANKL, M-CSF, or VEGF over time when MSCs and RAW264 cells were co-cultured (Fig. 5). On the other hand, expression of OPG was significantly $(P<0.01)$ decreased on day 6,8 , and 10 relative to day 4 . The expression level of OPG was lowest on day 8 , being only about $1 / 14$ of the level on day 4 .

\section{Amount of RANKL and OPG protein secreted into co-cultures of MSCs and RAW264 cells}

On day 8, lower levels of RANKL protein in medium from co-cultures of MSCs and RAW264 cells were observed, compared to MSCs cultured alone (0.188 [0.161-0.194] $\mathrm{pg} / \mathrm{mL}$ vs. 0.249 [0.210-0.262] pg/mL, respectively, $P$ $<0.05$ ) (Fig. 6). The amount of OPG protein secreted into medium of co-cultured MSCs and RAW264 cells was significantly higher than that in medium of MSCs cultured alone (249 [244-258] pg/mL vs. 147 [142-165] $\mathrm{pg} / \mathrm{mL}$, respectively, $P<0.01$ ). In addition, the amount of 
OPG protein was obviously higher than that of RANKL protein.

\section{Discussion}

Bone regeneration is an important factor for successful repair of cleft lip and palate. Although regenerative medicine using MSCs and carriers has received attention, few attempts have been made to apply this clinically. Because the involvement of osteoclasts and transplanted MSCs in carrier bone resorption is unclear, the effect of MSCs on osteoclasts was investigated. For bone remodeling by osteoblasts and osteoclasts, cytokine-mediated interactions and signal transduction via cell-cell contact are considered necessary (12). In addition, Mbalaviele et al. (13) have reported that MSCs promote osteoclast differentiation by direct contact with CD34-positive hematopoietic stem cells.

In the present study, the time course of RANKL, $\mathrm{M}-\mathrm{CSF}$, and VEGF expression in MSCs cultured alone peaked at day 6 and 8 . On the other hand, enhancement of OPG expression was observed from day 4 to day 10 . Moreover, osteoclast differentiation was observed on the 2nd day in the presence of sRANKL when RAW264 cells were cultured alone. It was confirmed that the sRANKL concentration in the culture solution was sufficient for differentiation.

This data revealed that the level of expression of OPG protein was clearly higher than that of RANKL protein. These observations suggest that the expression level of RANKL in MSCs was not significantly involved in osteoclast differentiation. In co-culture with MSCs, osteoclast differentiation was not observed on day 4, and the number of osteoclasts was significantly decreased in comparison with RAW264 cells cultured alone. Although the expression level of RANKL in MSCs cultured alone was high on day 8 , co-culture with MSCs significantly suppressed the expression of the osteoclast differentiation transcription factors NF- $\kappa$ B and NFATc1. These results suggested that MSC-derived factor(s) inhibited osteoclast differentiation in RAW264 cells during co-culture. Chanda et al. $(15,16)$ have reported constitutive OPG production by MSCs. Using a cytokine antibody array and MSC-conditioned medium, Ando et al. (17) found that OPG production by MSCs was relatively high. In addition, Nelson et al. (18) reported that the affinity of OPG for RANKL was higher than that for RANK. Accordingly, it was speculated that OPG, a decoy receptor for RANKL produced by MSCs, became bound to sRANKL and inhibited osteoclast differentiation. In an indirect co-culture experiment using human MSCs and peripheral blood mononuclear cells, Chanda et al. $(15,16)$ found that production of OPG suppressed the differentiation of osteoclasts. A similar result was obtained in the present study. However, it was reported that osteoclast differentiation was restored by OPG knockdown with siRNA or addition of OPG neutralizing antibody, and that expression of cathepsin K, a marker of osteoclasts, was also increased (16). Because addition of these antibodies does not completely restore osteoclast differentiation, interleukin-10 and prostaglandin $E_{2}$ may also be involved $(19,20)$. Tumor necrosis factor- $\alpha$ and other molecules may be involved in the osteoclast differentiation pathway (21), suggesting that osteoclast differentiation may be inhibited through other routes. These possibilities await further investigation.

OPG production by MSCs during co-culture decreased over time, and this was considered to explain the gradual osteoclast differentiation that was observed beginning on day 6. During co-culture with RAW264 cells, a feedback mechanism may have been operating, and cell function may have decreased due to cell fatigue.

Maes et al. (22) reported that early vessel entry is essential for bone formation at fracture sites. Similarly, angiogenesis prior to bone regeneration is also considered indispensable for successful transplantation. In the present study, no significant temporal change in the expression of VEGF during co-culture was observed. Chen et al. (23) reported that MSCs constitutively secrete a large amount of VEGF protein, and King et al. (24) observed an increase in VEGF in MSCs during indirect co-culture with macrophages. Oskowitz et al. (25) also reported that expression of VEGF in MSCs increases in the absence of serum. In addition, Kim et al. (26) reported that VEGF, which is thought to play an important role in bone remodeling, increases the expression of RANKL in synovial fibroblasts.

The results of this study revealed that MSCs inhibited osteoclast differentiation of RAW264 cells by suppressing the expression of factors that induce the process. MSCs may also affect the differentiation of osteoclasts via expression of OPG. It has been reported that transplantation of MSCs to increase bone regeneration significantly increased the number of differentiated osteoclasts beginning at 4 weeks after transplantation (27), and that absorption of carriers was promoted in the long term (4). Although it is suspected that MSCs are involved in osteoclast differentiation, these findings suggested that MSCs inhibited osteoclast differentiation. Therefore, it seems that further clarification of this complex phenomenon is needed. It was suggested that indirect co-culture with MSCs inhibits osteoclast differentiation, creating a favorable environment for bone regeneration. 


\section{Acknowledgements}

This work was funded by a grant from the JSPS KAKENHI (No. 15K20595).

\section{Conflict of interest}

The authors have no conflict of interest to declare.

\section{References}

1. Natsume N, Suzuki T, Kawai T (1987) The prevalence of cleft lip and palate in the Japanese: their birth prevalence in 40,304 infants born during 1982. Oral Surg Oral Med Oral Pathol 63, 421-423.

2. Boyne PJ, Sands NR (1972) Secondary bone grafting of residual alveolar and palatal clefts. J Oral Surg 30, 87-92.

3. Gimbel M, Ashley RK, Sisodia M, Gabbay JS, Wasson KL, Heller J et al. (2007) Repair of alveolar cleft defects: reduced morbidity with bone marrow stem cells in a resorbable matrix. J Craniofac Surg 18, 895-901.

4. Tanimoto K, Yanagida T, Tanne K (2010) Orthodontic treatment with tooth transplantation for patients with cleft lip and palate. Cleft Palate Craniofac J 47, 499-506.

5. Yoshioka M, Tanimoto K, Tanne Y, Sumi K, Awada T, Oki N et al. (2012) Bone regeneration in artificial jaw cleft by use of carbonated hydroxyapatite particles and mesenchymal stem cells derived from iliac bone. Int J Dent 2012, 1-8.

6. Hattersley G, Kerby J, Chambers TJ (1991) Identification of osteoclast precursors in multilineage hemopoietic colonies. Endocrinology 128, 259-262.

7. Quinn JM, Neale S, Fujikawa Y, McGee JO, Athanasou NA (1998) Human osteoclast formation from blood monocytes, peritoneal macrophages, and bone marrow cells. Calcif Tissue Int 62, 527-531.

8. Khosla S (2001) Minireview: the OPG/RANKL/RANK system. Endocrinology 142, 5050-5055.

9. Martin TJ, Sims NA (2005) Osteoclast-derived activity in the coupling of bone formation to resorption. Trends Mol Med 11, 76-81.

10. Boyle WJ, Simonet WS, Lacey DL (2003) Osteoclast differentiation and activation. Nature 423, 337-342.

11. Takahashi N, Akatsu T, Udagawa N, Sasaki A, Yamaguchi A, Moseley MJ et al. (1988) Osteoblastic cells are involved in osteoclast formation. Endocrinology 123, 2600-2602.

12. Jimi E, Nakamura I, Amano H, Taguchi Y, Tsurukai T, Tamura M et al. (1996) Osteoclast function is activated by osteoblastic cells through a mechanism involving cell-to-cell contact. Endocrinology 137, 2187-2190.

13. Mbalaviele G, Jaiswal N, Meng A, Cheng L, Van Den Bos C, Thiede M (1999) Human mesenchymal stem cells promote human osteoclast differentiation from CD34+ bone marrow hematopoietic progenitors. Endocrinology 140, 3736-3743.

14. Bloemen V, Schoenmaker T, Vries T, Everts V (2010) Direct cell-cell contact between periodontal ligament fibroblasts and osteoclast precursors synergistically increases the expression of genes related to osteoclastogenesis. J Cell Physiol 222, 565-573.
15. Chanda D, Isayeva T, Kumar S, Hensel JA, Sawant A, Ramaswamy G et al. (2009) Therapeutic potential of adult bone marrow-derived mesenchymal stem cells in prostate cancer bone metastasis. Clin Cancer Res 15, 7175-7185.

16. Oshita K, Yamaoka K, Udagawa N, Fukuyo S, Sonomoto K, Maeshima K et al. (2011) Human mesenchymal stem cells inhibit osteoclastogenesis through osteoprotegerin production. Arthritis Rheum 63, 1658-1667.

17. Ando Y, Matsubara K, Ishikawa J, Fujio M, Shohara R, Hibi $\mathrm{H}$ et al. (2014) Stem cell-conditioned medium accelerates distraction osteogenesis through multiple regenerative mechanisms. Bone 61, 82-90.

18. Nelson CA, Warren JT, Wang M, Teitelbaum SL, Fremont DH (2012) RANKL employs distinct binding modes to engage RANK and the osteoprotegerin decoy receptor. Structure 20, 1971-1982.

19. Take I, Kobayashi Y, Yamamoto Y, Tsuboi H, Ochi T, Uematsu S et al. (2005) Prostaglandin E2 strongly inhibits human osteoclast formation. Endocrinology 146, 5204-5214.

20. Mohamed SG, Sugiyama E, Shinoda K, Taki H, Hounoki H, Abdel-Aziz HO et al. (2007) Interleukin-10 inhibits RANKLmediated expression of NFATc1 in part via suppression of c-Fos and c-Jun in RAW264.7 cells and mouse bone marrow cells. Bone 41, 592-602.

21. Hounoki H, Sugiyama E, Mohamed SG, Shinoda K, Taki $\mathrm{H}$, Abdel-Aziz $\mathrm{HO}$ et al. (2008) Activation of peroxisome proliferator-activated receptor gamma inhibits TNF-alphamediated osteoclast differentiation in human peripheral monocytes in part via suppression of monocyte chemoattractant protein-1 expression. Bone 42, 765-774.

22. Maes C, Kobayashi T, Selig M, Torrekens S, Roth S, Mackem $S$ et al. (2010) Osteoblast precursors, but not mature osteoblasts, move into developing and fractured bones along with invading blood vessels. Dev Cell 19, 329-344.

23. Chen L, Tredget EE, Wu PY, Wu Y (2008) Paracrine factors of mesenchymal stem cells recruit macrophages and endothelial lineage cells and enhance wound healing. PLoS One 3, e1886.

24. Lee HW, Jeon YH, Hwang MH, Kim JE, Park TI, Ha JH et al. (2013) Dual reporter gene imaging for tracking macrophage migration using the human sodium iodide symporter and an enhanced firefly luciferase in a murine inflammation model. Mol Imaging and Biol 15, 703-712.

25. Oskowitz A, Mcferrin H, Gutschow M, Carter ML, Pochampally R (2011) Serum-deprived human multipotent mesenchymal stromal cells (MSCs) are highly angiogenic. Stem Cell Res 6, 215-225.

26. Kim HR, Kim HH, Lee SH (2014) VEGF promotes RANKL expression and osteoclastogenesis in rheumatoid arthritis. Ann Rheum Dis 73, 792.

27. Gamblin AL, Brennan MA, Renaud A, Yagita H, Lézot F, Heymann D et al. (2014) Bone tissue formation with human mesenchymal stem cells and biphasic calcium phosphate ceramics: the local implication of osteoclasts and macrophages. Biomaterials 35, 9660-9667. 